\title{
THE CONDITION FOR A PFAFFIAN SYSTEM IN INVOLUTION $\dagger$
}

BY J. M. THOMAS

1. Introduction. If a pfaffian system is to be employed for solving a system of partial differential equations, an integral variety on which a given set of variables are independent is desired. A pfaffian system having a non-singular integral variety of this type is said to be in involution with respect to the variables in question. A necessary and sufficient condition for a linear pfaffian system in involution has been stated by Cartan in terms of what he calls the prolonged system. $\ddagger$ The present paper gives to the condition an alternative form which is obtained directly from the original system. The condition is here derived for generalized (that is, non-linear) systems. $\$$ The paper ends ( $\$ 3$ ) with a few remarks about singular integral varieties.

2. The Condition. The basis of the following treatment is a theorem, which is an immediate consequence of known results and for which a simple, direct proof can also be given. It may be regarded as the basic theorem in the theory of linear, homogeneous equations. It is: $A$ system of linear, homogeneous equations has a solution in which a specified set of unknowns can be given arbitrary values if and only if the rank of its matrix is unaltered by the omission of the columns corresponding to those unknowns. For the application of the theorem, we note that having a solution corresponding to arbitrary values of the given unknowns is equivalent to having a solution when each of the following sets of values is assigned to those unknowns:

$(1,0, \cdots, 0), \quad(0,1, \cdots, 0), \cdots, \quad(0,0, \cdots, 1)$.

Let the generalized pfaffian system be

$$
\omega^{\lambda}=0, \quad(\lambda=1,2, \cdots, \rho),
$$

$\dagger$ Presented to the Society under a different title, December 1, 1933.

$\ddagger$ E. Cartan, Annales de l'École Normale, (3), vol. 21 (1904), pp. 153-175.

$\S \mathrm{J}$. M. Thomas, An existence theorem for generalized pfaffian systems, this Bulletin, vol. 40, pp. 309-315. This paper will be cited as G. The reader is assumed to be familiar with its contents. 
and let the systems employed in effecting its solution $\dagger$ be denoted by $\Sigma_{\alpha}$.

The conditions that (2) have an integral variety, on which

$$
x^{i_{1}}, x^{i_{2}}, \cdots, x^{i_{k}}
$$

are independent, are found by putting

$$
x^{i_{j}}=u^{j}, \quad(j=1,2, \cdots, k) .
$$

$\Sigma_{\alpha}$ becomes thereby a non-homogeneous system $\Sigma_{\alpha}^{*}$, whose matrix $M_{\alpha}^{*}$ can be obtained from $M_{\alpha}$ by omitting from it the columns corresponding to (3) and making the substitution (4) in the rest.

Systems $\Sigma_{1}^{*}, \cdots, \Sigma_{k}^{*}$ also arise if the equations (2) are regarded as identities in the differentials of (3), the variables other than (3) being regarded as functions of (3). For this reason, $\Sigma_{1}^{*}, \cdots, \Sigma_{k}^{*}$ will be said to arise by parametrizing (2) with respect to (3). Let $\Sigma_{2}^{*}$ become $\bar{\Sigma}_{2}^{*}$ when a general solution of $\Sigma_{1}^{*}$ has been substituted in it, etc. The characters of $M_{1}^{*}$, $\bar{M}_{2}^{*}, \cdots, \bar{M}_{k+1}^{*}$ will be called the characters of the parametrized system.

Every system $\Sigma_{\alpha},(\alpha=1,2, \cdots, k)$, contains the set of linear equations $[\alpha]=0$, whose matrix is $M_{1}$ for all values of $\alpha$ and which coincides with $\Sigma_{1}$ for $\alpha=1$. The substitution of (4) in $\Sigma_{1}, \cdots, \Sigma_{k}$ shows, therefore, that the algebraic system $\Sigma_{1}$ must have a solution in which the unknowns $x_{1} i_{1}, \cdots, x_{k}{ }^{i_{k}}$ have any set of values in (1). The fundamental theorem above shows that $M_{1}$ and $M_{1}^{*}$ must have the same character, if a solution exists.

Every system $\Sigma_{\alpha},(\alpha=2,3, \cdots, k)$, contains the set of equations $[\alpha]=0,[1 \alpha]=0$, whose matrix is $M_{2}$ and which coincides with $\Sigma_{2}$ for $\alpha=2$. Hence it follows that $\bar{\Sigma}_{2}$ must have a solution in which the unknowns $x_{2}{ }^{i_{1}}, \cdots, x_{2}{ }^{i_{k}}$ have any set of values from the last $k-1$ lines of (1). We have already seen that $[\alpha]=0$ must have a solution corresponding to the first line. That $[1 \alpha]=0$ must also, is seen by choosing $x_{\alpha}{ }^{i}=x_{1}{ }^{i}$ and remarking that $x_{1} i_{1}, \cdots, x_{1} i_{k}$ have the values of the first line of (1) in the solution (4).

The reasoning can be continued to include the matrix $\bar{M}_{k+1}^{*}$,

$\dagger \mathrm{G}$, equations (10). 
which arises from $\Sigma_{k+1}$ and which must be considered in order to insure non-singularity. A necessary condition for the existence of a non-singular variety is accordingly that the two sequences $M_{1}{ }^{*}, \bar{M}_{2}{ }^{*}, \cdots, \bar{M}_{k+1}^{*}$ and $M_{1}, \bar{M}_{2}, \cdots, \bar{M}_{k+1}$ have the same characters.

The condition is also sufficient. The process previously developed $\dagger$ serves to construct the integral variety, provided we put $u^{\alpha+1}, \cdots, u^{k}$ equal to their non-singular initial values, which are possibly different from zero, before determining the solution of $\bar{\Sigma}_{\alpha}^{*}$ by Cauchy's theorem. Because of (4), the rank of the functional matrix $J_{k}$ is $k$. If $k<\gamma$, we may continue, and obtain an integral variety of higher dimension having the desired property.

THEOREM. A generalized pfaffian system has a non-singular integral variety on which $k$ specified variables are independent if and only if its first $k+1$ characters are unaltered by parametrization with respect to those variables written in some order.

An example is furnished by the equation

$$
x^{2} d x^{1}+x^{1} d x^{2}+x^{3} d x^{4}=0,
$$

for which $m_{1}=1, m_{2}=m_{3}=2$. Parametrizing with respect to $x^{1}, x^{2} ; x^{1}, x^{3}$, we get the characters $1,2,2 ; 1,1,1$, respectively. Hence there is a non-singular integral variety on which $x^{1}, x^{2}$ are independent, but none on which $x^{1}, x^{3}$ are.

3. Singular Integral Varieties. The integration of the system of partial differential equations

$$
\frac{\partial z}{\partial x}=1, \quad \frac{\partial z}{\partial y}=\frac{z}{x+y}
$$

is equivalent to finding an integral variety on which $x$ and $y$ are independent for the pfaffian system

$$
(x+y) d x+z d y-(x+y) d z=0 .
$$

Since $\gamma=1$, the integral variety sought, if it exists, must be singular and is not furnished by the above theorem. On the other hand, Riquier's method of reduction to passive orthonomic form applied to (5) gives immediately the solution $z=x+y$.

$\dagger \mathrm{G}, \S 2$. 
Burstin $\dagger$ has recognized the essential element of Cartan's method and applied it in an attempt to obtain all integral varieties for the linear case. To determine an integral variety of dimension $\sigma$ he finds the finite conditions $F$ which the variables must satisfy in order that the algebraic equations in $x_{\alpha}{ }^{i}$ have $\sigma$ independent solutions. Elimination of some of the $x$ 's by means of $F$ gives a system in the others. His treatment of the resulting system seems defective. We shall employ the notation of the present paper in describing his results. He replaces the nonsingular integral variety $V_{k}$ by any integral variety $W_{k}$, that is, by any functions of $u^{1}, \cdots, u^{k}$ satisfying

$$
\Sigma_{1}+\Sigma_{2}+\cdots+\Sigma_{k} \text {. }
$$

He then determines a variety $W_{k+1}$ passing through $W_{k}$ by applying Cauchy's theorem to a solved form of $\Sigma_{k+1}$. He does not state $\ddagger$ explicitly the manner of solving $\Sigma_{k+1}$, but it must be solved in the presence of (7) if all integral varieties are to be obtained. His proof that $W_{k+1}$ satisfies (7) for all values of $u^{k+1}$ is vitiated by the assumption that it satisfies $\Sigma_{k+1}$, some of whose equations it may only satisfy by virtue of (7). Hence $W_{k+1}$ may not be integral. This is illustrated by the following example:

$$
d x^{1}+x^{3} d x^{4}+x^{3} d x^{5}=0, d x^{2}+x^{4} d x^{5}+x^{6} d x^{7}=0 .
$$

We find $m_{1}=2, m_{2}=4$, and $F$ is the whole of space, if a variety of dimension two is sought. An algebraic solution of $\Sigma_{1}$ is

$$
x_{1}^{1}=0, \quad x_{1}^{2}=x^{4} x_{1}^{4}-x^{6} x_{1}^{7}, \quad x_{1}^{3}=0, \quad x_{1}^{5}=-x_{1}^{4} .
$$

The introduction of these values in $\Sigma_{2}$ gives

$$
\begin{gathered}
x_{2}^{1}+x^{3} x_{2}^{4}+x^{3} x_{2}^{5}=0, \quad x_{2}^{2}+x^{4} x_{2}^{5}+x^{6} x_{2}^{7}=0, \\
x_{1}^{4} x_{2}^{4}+x_{1}^{4} x_{2}^{5}+x_{1}^{6} x_{2}^{7}-x_{2}^{6} x_{1}^{7}=0 .
\end{gathered}
$$

A solution of (9) is furnished by

$$
f^{1}=f^{2}=f^{3}=0, \quad f^{4}=f^{6}=f^{7}=u, \quad f^{5}=-u .
$$

A solution of (10) reducing to (11) for $v=0$ is

†C. Burstin, Recueil Mathématique de la Société Mathématique de Moscou, vol. 37 (1930), pp. 13-21.

$\ddagger$ See his formulas (33), (34). 


$$
\begin{aligned}
& \phi^{1}=-u^{2} v^{2}, \quad \phi^{2}=-u^{3} v+\frac{1}{2} u^{4} v^{2}, \quad \phi^{3}=v, \quad \phi^{4}=u, \\
& \phi^{5}=-u+2 u^{2} v, \quad \phi^{6}=u+u^{2} v, \quad \phi^{7}=u-u^{2} v,
\end{aligned}
$$

where the superscripts on the parameters $u$ and $v$ denote powers. These values do not give an integral variety. They do not satisfy the first equation of $\Sigma_{1}$ for all values of $v$ because they fail to satisfy $\Sigma_{2}$.

By employing $\bar{\Sigma}_{k+1}$ and using relations like G (22), we prove (7) is satisfied, and Burstin's manner of passing a $W_{k+1}$ through a $W_{k}$ can be rendered valid, but it then gives, in general, only non-singular integral varieties and certain singular integral varieties of dimension exceeding $\gamma$ which owe their singularity to the satisfaction of relations involving the $x$ 's but not their derivatives, and which are obtained thanks to the preparatory elimination process he employs. Equation (6) furnishes a somewhat trivial example of the case in which his method is successful. The variety $F$ is $z=x+y$.

In general, the determination of singular integral varieties characterized by relations involving the derivatives of the $x$ 's requires the discussion of integrability conditions other than those expressed by the derived forms $\omega^{\prime}$. System (8) illustrates this also. If an integral variety on which

$$
x_{1}^{4}+x_{1}^{5}=0
$$

is desired, the last equation of (10) leads to the rather obvious condition $A_{1}=0$, where

$$
A=\left(x_{1}^{6} x_{2}^{7}-x_{2}^{6} x_{1}^{7}\right) / x_{1}^{4} .
$$

If in the method of the former paper $\mathrm{G}$, we replace $V_{k}$ by any integral variety of dimension $k$, and the general algebraic solution of $\bar{\Sigma}_{k+1}$ employed there by any particular holomorphic solution of $\Sigma_{k+1}$ for $x_{k+1}^{i}$, and in other respects proceed as before, we obtain a variety which is surely integral, and which may be singular or non-singular, but we cannot obtain all integral varieties in this manner.

Some of the singular integral varieties of a pfaffian system appear as non-singular integral varieties of an associated pfaffian system of higher degree. This application of the existence theorem will be discussed elsewhere.

Duke University 